\title{
布朗斯特酸促进的呋喃[2,3-b]喹啉类并环化合物的合成
}

\author{
张志国* 郑丹麻娜娜毕晶晶
}

(河南师范大学化学化工学院 新乡 453007)

\begin{abstract}
摘要 呋喃并埕啉结构单元广泛存在于很多天然产物中. 介绍一种在加热的条件下, 三氟甲磺酸促进的, 通过多取代 呋喃衍生物分子内环合反应制备呋喃[2,3-b]喹啉类化合物的方法. 该方法具有操作简单、官能团兼容性好、区域选择 性好和产品产率较高等优点.
\end{abstract}

关键词 布朗斯特酸; 呋喃 $[2,3-b]$ 喹啉; 三氟甲磺酸; 傅-克反应

\section{Brønsted Acid-Promoted the Synthesis of Furo[2,3-b]quinolines}

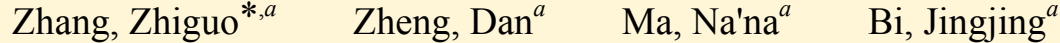 \\ $\left({ }^{a}\right.$ School of Chemistry and Chemical Engineering, Henan Normal University, Xinxiang 453007)
}

\begin{abstract}
The furoquinoline unit is present in many natural products. Here, an approach is presented for the preparing of furo[2,3-b]quinolines from readily available multi-substituted furans in the presence of Brønsted acid via an intramolecular cyclization under the heating conditions. Simple operation, good compatibility, high regioselectivity and morderate yields are the advantages of the method.

Keywords Brønsted acid; furo[2,3-b]quinoline; trifluoromethanesulfonic acid; Friedel-Crafts reaction
\end{abstract}

呋喃[2,3-b]喹啉类化合物是一种重要的含氮和氧杂 原子的三并杂环类化合物 ${ }^{[1]}$. 含有这类母核结构的生物 碱, 如白鲜碱、崖椒碱和吴英春碱等, 广泛存在于芸香 科植物中 ${ }^{[2]}$. 它们拥有多种生物活性，比如抗菌、抗病 毒、抗人类免疫缺陷病毒、抗炎、抗肿瘤和抗细胞毒素 等(图 1$)^{[3]}$. 白鲜碱是含有呋喃[2,3-b]喹啉结构单元的结 构最简单的呋喃并喹啉类天然产物. 1956 年, 呋喃并喹 啉类化合物的合成方法首次在德国《应用化学》杂志上 发表 ${ }^{[4]}$, 从此, 这类化合物的合成方法一直是有机合成 化学的热点研究领域 ${ }^{[5]}$. 目前, 文献报道的呋喃 [2,3-b] 喹啉类化合物的合成方法大致可以分为两种合成策略: (a)通过 3 位官能化喹啉衍生物环合反应合成呋喃 $[2,3-b]$ 喹啉类化合物. 这类反应一般是先在喹啉的 3 位构建一 个端位带有炔基、羟基, 烯基或者卤素等官能团的烷基 链, 然后再通过分子内环合反应得到呋喃[2,3- $b$ ] 喹啉类 化合物的呋喃部分(Scheme 1, a $)^{[6]}$. (b)通过官能化的呋<smiles>COc1c2ccccc2nc2occc12</smiles>

Dictamnine<smiles>COc1ccc2c(OC)c3c(nc2c1O)OCC3</smiles><smiles>OCC=C1COC2=C(CC2)C1</smiles>

Confusameline<smiles>COc1cc2nc3occc3c(OC)c2cc1OC</smiles>

图 1 几种代表性的含有呋喃[2,3-b]喹啉母核结构的天然产物 Figure 1 Several representative furo[2,3-b]quinolines-containing natural products

喃衍生物环合反应合成呋喃 $[2,3-b]$ 喹啉类化合物. 这类 反应一般是以亚胺和富电子的呋喃为原料, 通过杂 Diels-Alder 反应先构建一个角型的呋喃并喹啉类前体

\footnotetext{
* Corresponding author. E-mail: zhangzg@htu.edu.cn

Received December 8, 2016; revised January 19, 2017; published online March 1, 2017.

Project supported by the National Natural Science Foundation of China (Nos. 21272057, 21372065), the Young Backbone Teachers Fund of Henan Province (No. 2014GGJS-049), the Key Project of Henan Provincal Educational Committee (Nos. 14A150019, 14B150042, 15A150015), the Science \& Technology Innovation Talents in Universities of Henan Province (No. 17HASTIT002), and the Outstanding Young Talent Cultivation Project Funding of Henan Normal University (No. 14YR002).

国家自然科学基金(Nos. 21272057, 21372065)、河南省高等学校青年骨干教师资助计划(No. 2014GGJS-049)、河南省教育厅重点研究(Nos. 14A150019, 14B150042, 15A150015)、河南省高校科技创新人才支持计划(No. 17HASTIT002)、河南师范大学杰出青年人才培育基金(No. 14YR002)资助项目
} 
化合物, 然后再经过呋喃部分的开环再环合反应得到呋 喃[2,3-b]喹啉类化合物(Scheme 1, b $)^{[7]}$. 虽然通过上述 两种策略都可以获得呋喃[2,3-b]喹啉类化合物, 但是, 一些方法存在普适性差、区域选择性差(自由基环合反 应)、原料合成局限性大、反应条件苛刻以及所用试剂 昂贵等问题, 影响了这些反应的实用价值. 因此, 发展 新的呋喃并喹啉化合物的合成方法仍然具有重要的现 实意义和理论研究价值.

(a) 通过3位官能化喹啉衍生物环合反应合成呋喃[2,3- $b]$ 喹啉 类化合物

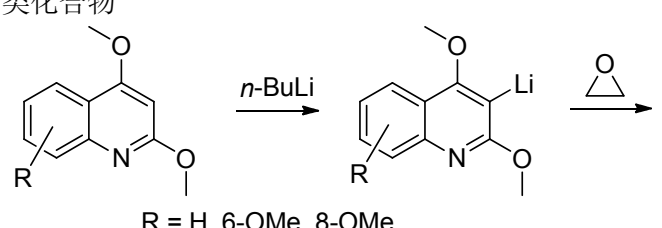
$\mathrm{R}=\mathrm{H}, 6-\mathrm{OMe}, 8-\mathrm{OMe}$<smiles>[R]c1cccc2c(OC)c(CCO)c(OC)nc12</smiles>

(b)通过官能化的呋喃衍生物环合反应合成呋喃[2,3- $b$ 喹啉 类化合物

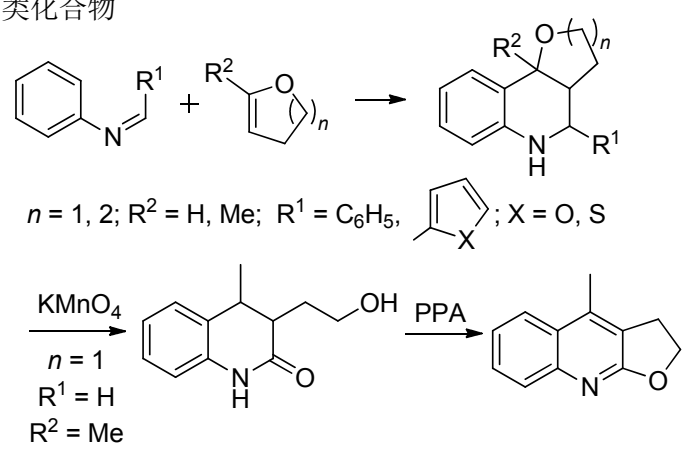

图式 1 常见的呋喃[2,3-b]喹啉类化合物的合成策略 Scheme 1 Strategies for the synthesis of furan[2,3-b]quinolines

串联反应因具有高效和实用的特点被广泛应用于 有机合成化学 ${ }^{[8]}$. 据我们所知, 通过同时构建呋喃环和 喹啉环的串联反应策略合成呋喃[2,3-b]喹啉类化合物的 文献报道不多 ${ }^{[5 a, 9]}$. 2016 年, Shi 等 ${ }^{[5 a]}$ 以邻氨基芳基亚烷 基环丙烷衍生物为原料, 在三光气(BTC)和三乙胺的联 合催化下, 通过形式上的分子内 $[3+2]$ 环加成串联反应 合成了呋喃 $[2,3-b]$ 喹啉类化合物(Eq. 1). 2013 年, Ru 等 ${ }^{[9 b]}$ 发现在卤化铜的作用下，1-酰基-1-芳酰胺基环丙烷 类化合物可以发生高选择性的开环加成反应, 生成一种 双卤代的 $\beta$-羰基酰胺类化合物, 这种酰胺衍生物可以在 硫酸的促进下进一步得到呋喃 [2,3- $b$ ] 喹啉类化合物 (Scheme 2). 2007 年, 我们课题组 ${ }^{[9 a}$ 利用高效的串联反 应, 通过先构建呋喃再构建喹啉环的新串联反应策略, 从 1-酰基-1-芳酰胺基环丙烷类化合物出发, 在五水四 氯化锡的催化下，经过分子内开环/再环合反应，合成了
一系列呋喃[2,3-b]喹啉类衍生物(Eq. 2). 同年，利用便 宜易得的双活化的 1-酰基-1-芳酰胺基环戊烷衍生物, 在硫酸的促进下, 合成了吡喃 $[2,3-b]$ 喹啉类生物碱 ${ }^{[10]}$. 该反应具有原料易得、产物产率高、反应普适性好和易 于操作等优点(Eq. 3). 在上述研究工作的基础 上 $^{[11]}$, 我们希望能够发展更多的合成呋喃 $[2,3-b]$ 喹啉类化合物 的新方法, 以满足这类化合物取代基多样性需要. 本文 介绍一种由简单易得的多取代呋喃为原料，在三氟甲磺 酸 $\left(\mathrm{CF}_{3} \mathrm{SO}_{3} \mathrm{H}\right)$ 的促进下，经过分子内亲电环合反应一步 生成呋喃 $[2,3-b]$ 喹啉类化合物的方法(Eq. 4).<smiles>[R]C(=C1CC1)c1c([R])cccc1N</smiles><smiles></smiles><smiles>[R]c1c2c(nc3ccccc13)OCC2</smiles>

图式 $2 \mathrm{Ru}$ 等合成呋喃[2,3-b]喹啉类化合物的工作 Scheme $2 \mathrm{Ru}$ et al. developed the synthesis of furo[2,3-b]quinolines<smiles>[R]C(=O)C1(C(=O)Nc2ccc([R7])c([R])c2[R])CC1[R]</smiles><smiles>[R]c1cccc(NC(=O)C2(C(C)=O)CCCC2)c1</smiles><smiles>[R1]C(=O)c1cc(C)oc1Nc1cccc([R])c1</smiles>

\section{1 结果与讨论}

首先，以 $N, N$-二甲基甲酰胺(DMF)为溶剂，在无水 $\mathrm{K}_{2} \mathrm{CO}_{3}$ 的作用下, 以乙酰乙酰苯胺和 1,2,3-三溴丙烷为 原料，通过分子间 $C, O$-双烷基化反应制得了多取代的 呋喃衍生物 $\mathbf{1 a}^{[\mathrm{a}]}$. 在得到了化合物 $1 \mathrm{a}$ 以后, 我们开始 
从催化剂的种类、用量、反应温度及溶剂种类等几个方 面对该反应的条件进行了优化，尝试合成目标化合物 2a. 主要的实验结果总结在表 1 中. 2007 年, Klumpp 课 题组 ${ }^{[12]}$ 通过利用理论计算和核磁共振波谱等手段证明 三氟甲磺酸是促进酰基和芳烃发生傅一克反应最有效的 质子酸, 因此, 我们首先选择了三氟甲磺酸尝试促进该 环合反应. 经过多次尝试我们发现, 在 $80{ }^{\circ} \mathrm{C}$ 时, 在 8 倍 量的三氟甲磺酸中, $10 \mathrm{~h}$ 以后反应混合物经过中和, 萃 取, 柱层析分离提纯后, 环合产物 $\mathbf{2 a}$ 的产率可以达到 $76 \%$ (表 1, Entry 2). 如果降低或者升高反应的温度, 产 物 $2 \mathrm{a}$ 的产率都会降低, 只是在越高的温度下反应的速 度越快(表 1, Entries 1, 3). 增加三氟甲磺酸的用量到 10 倍量也不能提高产物 $\mathbf{2 a}$ 的产率(表 1, Entry 4). 另外, 我 们也尝试了使用低于 8 倍量的三氟甲磺酸促进这个反 应, 但是由于三氟甲磺酸的用量太少, 底物 $1 \mathrm{a}$ 不能很好 地溶解. 我们还尝试了其它的液体的布朗斯特酸, 比如: 质子酸多聚磷酸(PPA)、浓硫酸 $\left(\mathrm{H}_{2} \mathrm{SO}_{4}\right)$ 和路易斯酸三氟 甲磺酸三甲基硅酯(TMSOTf). 实验结果表明, 虽然 PPA 可以促进 $2 \mathbf{a}$ 的收率达到 $71 \%$, 但是反应时间需要 $24 \mathrm{~h}$ (表 1, Entry 5). 浓硫酸也需要 $14 \mathrm{~h}$ 才能得到 $68 \%$ 的 $2 \mathbf{a}$ (表 1, Entry 6). 虽然 TMSOTf 参与的反应速率较快, 但 是产物产率只能达到 57\% (表 1, Entry 7). 此外, 我们还 尝试了对甲苯磺酸 $(p-\mathrm{Ts} \mathrm{OH})$ 这种固体酸对该环合反应 的影响. 为了使固体的原料 $1 \mathrm{a}$ 和对甲苯磺酸溶解形成 均一的溶液, 我们使用了 $1 \mathrm{~mL}$ 的 1,4-二氧六环作溶剂,

表 1 优化条件表 ${ }^{a}$

Table 1 Survey of the reaction conditions

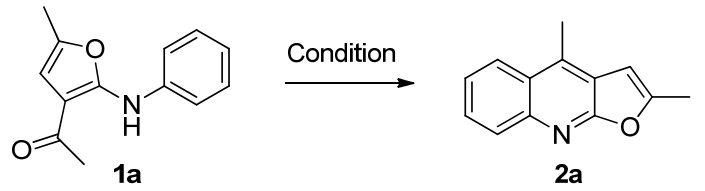

\begin{tabular}{clccc}
\hline Entry & Acid (equiv.) & $T /{ }^{\circ} \mathrm{C}$ & Time/h & Yield of $\mathbf{2 a} / \%$ \\
\hline 1 & $\mathrm{CF}_{3} \mathrm{SO}_{3} \mathrm{H}(8)$ & 60 & 14 & $59^{b}$ \\
2 & $\mathrm{CF}_{3} \mathrm{SO}_{3} \mathrm{H}(8)$ & 80 & 10 & 76 \\
3 & $\mathrm{CF}_{3} \mathrm{SO}_{3} \mathrm{H}(8)$ & 100 & 8 & 70 \\
4 & $\mathrm{CF}_{3} \mathrm{SO}_{3} \mathrm{H}(10)$ & 80 & 10 & 67 \\
5 & $\mathrm{PPA}(8)_{6}$ & 80 & 24 & 71 \\
6 & $\mathrm{Con} . \mathrm{H}_{2} \mathrm{SO}_{4}(8)$ & 80 & 14 & $68^{c}$ \\
7 & TMSOTf (8) & 80 & 6 & $57^{d}$ \\
8 & $p$-TsOH (8) & 80 & 10 & $0^{e}$ \\
9 & $\mathrm{AcOH}(8)$ & 80 & 10 & $0^{f}$ \\
10 & PivOH (8) & 80 & 10 & $0^{g}$ \\
11 & $\mathrm{TFA} \mathrm{(8)}$ & 80 & 10 & $0^{h}$ \\
12 & $\mathrm{TCA}(8)$ & 80 & 10 & $0^{i}$ \\
\hline
\end{tabular}

${ }^{a}$ 除非另外说明, 所有反应都是在空气氛围中进行, 反应物 1a 的用量为 $0.2 \mathrm{mmol}$, 收率为分离收率; ${ }^{b} 18 \%$ 的 $1 \mathrm{a}$ 被回收. ${ }^{c} 5 \%$ 的 $1 \mathrm{a}$ 被回收.

${ }^{d}$ TMSOTf 的沸点为 $77{ }^{\circ} \mathrm{C}$, 因此反应在密封管中进行. ${ }^{e} 20 \%$ 的 1a 被回收.

${ }^{f} 16 \%$ 的 1a 被回收. ${ }^{g} 30 \%$ 的 1a 被回收. ${ }^{h} 5 \%$ 的 1a 被回收. ${ }^{i} 12 \%$ 的 1a 被回收.
结果我们观察到反应产生了非常复杂的混合物(表 1 , Entry 8). 其它一些常见的质子酸, 如: 醋酸 $(\mathrm{AcOH})$ 、特 戊酸 $(\mathrm{PivOH}) 、$ 三氟乙酸(TFA)和三氯乙酸(TCA)在 $10 \mathrm{~h}$ 内不能得到出目标化合物(表 1, Entries 9 12).

在确定了最佳的反应条件以后(表 1, Entry 2), 我们 对反应的适用范围进行了研究, 主要结果总结归纳在表 2 中. 首先, 我们研究了各种 $\mathrm{R}^{2}$ 取代基对反应的影响. 实验表明，不但苯基取代的原料 $1 \mathrm{a}$ 可以顺利地得到目 标化合物 $2 \mathrm{a}$ (产率 76\%), 而且，当 $\mathrm{R}^{2}$ 取代基为甲氧基或 者甲基时，原料也可以顺利地生成相应的目标产物 2 . 邻甲氧基取代的原料 $1 \mathrm{~b}$ 可以以 $75 \%$ 的收率给出目标产 物 $2 \mathrm{~b}$. 邻位和对位甲基取代的底物 $1 \mathrm{c}$ 和 $1 \mathrm{e}$ 也可以分别 生成目标化合物 $2 \mathrm{c}$ 和 $2 \mathrm{e}$ (产率: $72 \%$ 和 77\%). 对于弱吸 电子的取代基 $\mathrm{R}^{2}$ ，如 $\mathrm{Cl}$ 和 $\mathrm{Br}$ 等卤代基团，反应 $\mathbf{1 f} \sim \mathbf{1 i}$ 也可以顺利的进行，邻、间和对位卤代的产物 $\mathbf{2 f} \sim \mathbf{2 i}$ 的 收率可以达到 73\% 79\%. 但是，苯基对位带有酯基这 种强吸电子基的原料 $\mathbf{1} \mathbf{j}$ 不能得到环合产物 $\mathbf{2} \mathbf{j}, 78 \%$ 的原 料 $1 \mathbf{j}$ 被回收. 这一结果符合傅一克反应适用范围所描述 的当芳基带有强吸电子基时傅-克反应不能发生这一结 论，因此硝基苯可以作为这类反应的溶剂 ${ }^{[13]}$. 而化合物

表 2 底物扩展 ${ }^{a}$

Table 2 Extension of the reaction scope<smiles>[R1]C(=O)c1cc(C)oc1Nc1cccc([R])c1</smiles><smiles>Cc1cc2c(C)c3ccccc3nc2o1</smiles>

2a, 10 h, $76 \%$<smiles>Cc1ccc2nc3oc(C)cc3c(C)c2c1</smiles>

2d, 8 h, $72 \%$<smiles>Cc1cc2c(C)c3ccc(Cl)cc3nc2o1</smiles>

2g, $10 \mathrm{~h}, 73 \%$<smiles>COc1cccc2c(C)c3cc(C)oc3nc12</smiles>

2b, 9 h, $75 \%$<smiles>Cc1cc2c(C)c3cccc(C)c3nc2o1</smiles>

2c, $10 \mathrm{~h}, 77 \%$<smiles>Cc1cc2c(C)c3cccc(Cl)c3nc2o1</smiles>

2f, $10 \mathrm{~h}, 75 \%$ 2e, 9.5 h, $77 \%$<smiles>Cc1cc2c(C)c3cc(Cl)ccc3nc2o1</smiles>

2h, 10 h, 79\%

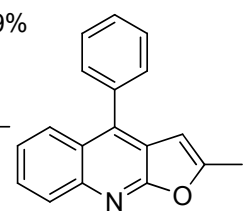

2i, $11 \mathrm{~h}, 79 \%$

2j, 12 h, $0 \%$

2k, 9 h, $91 \%$

$\bar{a}$ 除非特别指出, 所有反应物均使用 $\mathbf{1}(0.2 \mathrm{mmol}), \mathrm{CF}_{3} \mathrm{SO}_{3} \mathrm{H}(1.6 \mathrm{mmol})$, 反应温度为 $80{ }^{\circ} \mathrm{C}$, 收率为分离收率. 
1f 1i 能够发生该亲电环合反应的原因是氨基是带有供 电子性质的基团，它对傅-克反应的影响大于像卤素这 样的弱吸电子基. 还值得一提的是产物 $2 \mathbf{i}$, 溴取代基能 够被反应很好地兼容, 这有利于这类化合物进一步发生 Heck 反应等衍生化反应，从而得到更加有用的分子. 另 外, 由于取代基 $\mathrm{R}^{1}$ 的局限性, 我们只尝试了当 $\mathrm{R}^{1}$ 为苯 基取代基的原料是否能够发生这类反应. 实验结果表 明，化合物 $1 \mathbf{k}$ 可以顺利的发生亲电环合反应，得到 $91 \%$ 的目标产物 $\mathbf{2 k}$.

\section{2 反应机理的研究}

根据近些年的文献报道 ${ }^{[9 a, 10,14]}$ 和 Combes 喹啉类化 合物合成原理 ${ }^{[11]}$, 我们认为这个反应经历了分子内芳基 亲电环合芳构化的反应机理. 首先, 在加热和布朗斯特 酸的共同作用下, 多取代呋喃类化合物 1 的羰基上的氧 原子被质子化, 形成氧鎓离子中间体 $\mathbf{A}^{[12]}$, 然后中间体 $\mathbf{A}$ 发生分子内亲电环合反应生成中间体 $\mathbf{B}$, 最后, 再经 过质子转移和脱水反应得到呋喃[2,3-b]喹啉类化合物 2 (Scheme 3).

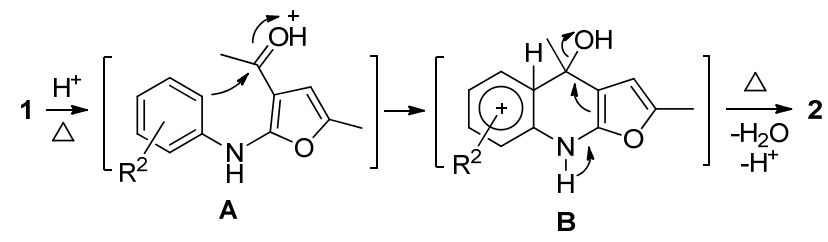

图式 3 反应可能的机理

Scheme 3 Proposed mechanism

\section{3 结论}

总之，发展了一种布朗斯特酸酸促进的以多取代呋 喃为原料一步合成具有重要意义的呋喃 $[2,3-b]$ 喹啉类化 合物的新方法. 该方法的原料便宜易得, 条件温和, 反 应易操作, 化学选择性和区域选择性好, 产物产率较高. 该方法进一步丰富了先构建杂环, 再形成喹啉环的合成 杂环并喹啉类生物碱的合成策略, 可以为设计与合成其 它的杂环并喹啉类化合物提供实验支持和理论指导.

\section{4 实验部分}

\section{1 仪器与试剂}

${ }^{13} \mathrm{C}$ NMR 和 ${ }^{1} \mathrm{H}$ NMR 由 Bruker DPX-400M 核磁共 振仪测定, 其中 TMS 作为内标, $\mathrm{CDCl}_{3}$ 或 DMSO- $d_{6}$ 作为 溶剂; HR-MS 由瑞士布鲁克公司高分辨率质谱测定仪: ESI 源测定; 化合物熔点是由巩义市予华仪器责任有限 公司的 X-5 显微熔点测定仪测定; TLC 检测使用巩义市 予华仪器责任有限公司 ZF-20D 暗箱式紫外分析仪; 化 合物称量使用奥豪斯国际贸易有限公司电子分析天平; 溶剂旋蒸使用巩义市予华仪器责任有限公司 YRE-2000 旋转蒸发仪; 高分辨质谱由 Bruker microToF II 高分辨 率质谱仪测定.

药品和试剂见表 3.

\section{2 化合物 2 的合成}

以 $2 \mathbf{b}$ 为例. 在 $25 \mathrm{~mL}$ 圆底烧瓶中加入 2-( $N$-邻甲氧 基苯基)-3-乙酰基-5-甲基呋喃(1b) $49 \mathrm{mg}$ (0.2 mmol)、三 氟甲磺酸 $0.14 \mathrm{~mL}(1.6 \mathrm{mmol})$, 在 $80{ }^{\circ} \mathrm{C}$ 油浴中加热反应

表 3 药品和试剂

Table 3 Starting materials and reagents

\begin{tabular}{|c|c|c|}
\hline 药品或试剂名称 & 纯度 & 生产厂家 \\
\hline 苯甲酰乙酸乙酯 & A.R. & 上海达瑞精细化学品有限公司 \\
\hline 对甲氧基苯甲酰乙酸乙酯 & A.R. & 梯希爱(上海)化成工业发展有限公司 \\
\hline 乙酰乙酸乙酯 & A.R. & 上海麦克林生化科技有限公司 \\
\hline 苯胺 & A.R. & 国药集团化学试剂有限公司 \\
\hline 对溴苯胺 & A.R. & 上海嘉辰 \\
\hline 对甲氧基苯胺 & A.R. & 阿拉丁 \\
\hline 乙酰乙酰苯胺 & A.R & 青岛双桃精细化工(有限)公司 \\
\hline 2-氯乙酰乙酰苯胺 & A.R. & 青岛双桃精细化工(有限)公司 \\
\hline 4-氯乙酰乙酰苯胺 & A.R. & 青岛双桃精细化工(有限)公司 \\
\hline 4-甲氧基乙酰乙酰苯胺 & A.R. & 青岛双桃精细化工(有限)公司 \\
\hline 2-甲氧基乙酰乙酰苯胺 & A.R & 青岛双桃精细化工(有限)公司 \\
\hline 4-甲基乙酰乙酰苯胺 & A.R. & 青岛双桃精细化工(有限)公司 \\
\hline 2-甲基乙酰乙酰苯胺 & A.R. & 青岛双桃精细化工(有限)公司 \\
\hline 2,4-甲基乙酰乙酰苯胺 & A.R. & 青岛双桃精细化工(有限)公司 \\
\hline 1,2,3-三溴丙烷 & A.R. & 阿拉丁 \\
\hline 无水碳酸钾 & A.R. & 国药集团化学试剂有限公司 \\
\hline$N, N$-二甲基甲酰胺 & A.R. & 成都市科龙化工试剂厂 \\
\hline 三氟甲磺酸 & A.R. & 阿拉丁 \\
\hline
\end{tabular}


混合物，直到原料 $\mathbf{1 b}$ 消失，反应过程使用 TLC 监测. 反 应结束后将反应混合物冷却至室温, 向反应混合物中加 入 $10 \mathrm{~mL}$ 水和 $1.5 \mathrm{~mL}$ 碳酸氢钠饱和溶液, 中和反应混合 物至中性, 有不溶固体析出, 抽滤, 水洗滤饼 $(2 \mathrm{~mL} \times 3)$, 得到的灰色固体粗产品经柱层析分离纯化 $[V($ 乙酸乙 酯 $) / V$ (石油醚) $=1 / 10]$, 得白色固体产物 8-甲氧基-2,4-二 甲基呋喃[2,3-b]喹啉(2b), $34 \mathrm{mg}$, 产率 75\%. m.p. 158 $160{ }^{\circ} \mathrm{C} ;{ }^{1} \mathrm{H}$ NMR $\left(400 \mathrm{MHz}, \mathrm{CDCl}_{3}\right) \delta: 7.68(\mathrm{~d}, J=8.4$, 1H), $7.51(\mathrm{~s}, 1 \mathrm{H}), 7.47 \sim 7.40(\mathrm{~m}, 1 \mathrm{H}), 7.06(\mathrm{~d}, J=7.6 \mathrm{~Hz}$, $1 \mathrm{H}), 4.10(\mathrm{~s}, 3 \mathrm{H}), 2.97$ (s, 3H), 2.47 (s, 3H); ${ }^{13} \mathrm{C}$ NMR $\left(100 \mathrm{MHz}, \mathrm{CDCl}_{3}\right) \delta: 143.2,126.9,124.0,119.9,115.4$, 115.1, 106.7, 56.0, 14.7, 11.5. HRMS (ESI) calcd for $\mathrm{C}_{14} \mathrm{H}_{13} \mathrm{NNaO}_{2}\left([\mathrm{M}+\mathrm{Na}]^{+}\right) 250.0838$, found 250.0842 .

2,4-二甲基呋喃[2,3-b]喹啉(2a): 白色固体, $30 \mathrm{mg}$, 产率 76\%. m.p. $127 \sim 129{ }^{\circ} \mathrm{C} ;{ }^{1} \mathrm{H}$ NMR $(600 \mathrm{MHz}$, $\left.\mathrm{CDCl}_{3}\right) \delta: 8.28(\mathrm{~d}, J=8.4 \mathrm{~Hz}, 1 \mathrm{H}), 8.21(\mathrm{~d}, J=8.4 \mathrm{~Hz}$, $1 \mathrm{H}), 7.88 \sim 7.85(\mathrm{~m}, 1 \mathrm{H}), 7.69 \sim 7.67(\mathrm{~m}, 1 \mathrm{H}), 7.60(\mathrm{~s}$, 1H), 3.09 (s, 3H), 2.53 (s, 3H); ${ }^{13} \mathrm{C}$ NMR (150 MHz, $\left.\mathrm{CDCl}_{3}\right) \delta: 158.6,143.6,131.4,126.1,125.4,124.1,120.9$, 116.3, 15.1, 11.2. HRMS (ESI) calcd for $\mathrm{C}_{13} \mathrm{H}_{12} \mathrm{NO}_{2}$ ([M+ H] ${ }^{+}$) 198.0913, found 198.0918.

2,4,8-三甲基呋喃[2,3- $b$ ]喹啉(2c): 白色固体, $32 \mathrm{mg}$, 产率 77\%. m.p. $110 \sim 112{ }^{\circ} \mathrm{C} ;{ }^{1} \mathrm{H}$ NMR $(400 \mathrm{MHz}$, $\left.\mathrm{CDCl}_{3}\right) \delta: 7.98(\mathrm{~d}, J=8.4 \mathrm{~Hz}, 1 \mathrm{H}), 7.57(\mathrm{~d}, J=6.8 \mathrm{~Hz}$, $1 \mathrm{H}), 7.50(\mathrm{~s}, 1 \mathrm{H}), 7.47 \sim 7.37(\mathrm{~m}, 1 \mathrm{H}), 2.99(\mathrm{~s}, 3 \mathrm{H}), 2.85$ (s, 3H), $2.48(\mathrm{~s}, 3 \mathrm{H}) ;{ }^{13} \mathrm{C}$ NMR (100 MHz, $\left.\mathrm{CDCl}_{3}\right) \delta$ : 161.0, 143.9, 142.6, 139.0, 136.5, 128.8, 125.8, 123.9, 121.5, 119.1, 115.3, 18.7, 14.5, 11.5. HRMS (ESI) calcd for $\mathrm{C}_{14} \mathrm{H}_{14} \mathrm{NO}\left([\mathrm{M}+\mathrm{H}]^{+}\right)$212.1070, found 212.1078.

2,4,6-三甲基呋喃[2,3- $b$ ]喹啉(2d): 白色固体，30 $\mathrm{mg}$, 产率 72\%. m.p. $135 \sim 137{ }^{\circ} \mathrm{C} ;{ }^{1} \mathrm{H}$ NMR $(400 \mathrm{MHz}$, $\left.\mathrm{CDCl}_{3}\right) \delta: 8.00 \sim 7.98(\mathrm{~m}, 1 \mathrm{H}), 7.79(\mathrm{~s}, 1 \mathrm{H}), 7.56 \sim 7.48$ (m, 1H), 7.44 (s, 1H), 2.89 (s, 3H), $2.56(\mathrm{~s}, 3 \mathrm{H}), 2.43$ (s, $3 \mathrm{H}) ;{ }^{13} \mathrm{C}$ NMR (100 MHz, $\left.\mathrm{CDCl}_{3}\right) \delta: 161.0,142.6,142.5$, $138.7,134.1,131.1,128.1,125.8,122.5,119.6,115.5$, 21.9, 14.3, 11.5. HRMS (ESI) calcd for $\mathrm{C}_{14} \mathrm{H}_{13} \mathrm{NNaO}$ $\left([\mathrm{M}+\mathrm{Na}]^{+}\right) 234.0889$, found 234.0890 .

2,4,6,8-四甲基呋喃[2,3- $b$ ] 喹啉(2e): 白色固体，36 $\mathrm{mg}$, 产率 77\%. m.p. 102 104 ${ }^{\circ} \mathrm{C} ;{ }^{1} \mathrm{H}$ NMR $(400 \mathrm{MHz}$, $\left.\mathrm{CDCl}_{3}\right) \delta: 7.68(\mathrm{~s}, 1 \mathrm{H}), 7.45(\mathrm{~s}, 1 \mathrm{H}), 7.39(\mathrm{~s}, 1 \mathrm{H}), 2.90(\mathrm{~s}$, $3 \mathrm{H}), 2.79$ (s, 3H), 2.52 (s, 3H), 2.44 (s, 3H); ${ }^{13} \mathrm{C}$ NMR $\left(100 \mathrm{MHz}, \mathrm{CDCl}_{3}\right) \delta: 160.4,142.1,137.8,135.8,133.0$, $130.9,125.5,120.1,118.7,115.0,99.7,21.6,18.4,14.2$, 11.3. HRMS (ESI) calcd for $\mathrm{C}_{15} \mathrm{H}_{16} \mathrm{NO}\left([\mathrm{M}+\mathrm{H}]^{+}\right)$ 226.1226, found 226.1229 .
8-氯-2,4-二甲基呋喃[2,3- $b$ ]喹啉(2f): 白色固体, 34 $\mathrm{mg}$, 产率 75\%. m.p. $143 \sim 145{ }^{\circ} \mathrm{C} ;{ }^{1} \mathrm{H}$ NMR $(600 \mathrm{MHz}$, $\left.\mathrm{CDCl}_{3}\right) \delta: 8.05(\mathrm{~d}, J=9.0 \mathrm{~Hz}, 1 \mathrm{H}), 7.83(\mathrm{~d}, J=7.2 \mathrm{~Hz}$, $1 \mathrm{H}), 7.55$ (s, 1H), $7.45 \sim 7.41(\mathrm{~m}, 1 \mathrm{H}), 3.00(\mathrm{~s}, 3 \mathrm{H}), 2.48$ (s, 3H); ${ }^{13} \mathrm{C}$ NMR $\left(150 \mathrm{MHz}, \mathrm{CDCl}_{3}\right) \delta: 161.8,143.6$, $141.2,139.6,132.7,128.8,127.2,124.0,122.7,120.4$, 115.3, 14.6, 11.4. HRMS (ESI) calcd for $\mathrm{C}_{13} \mathrm{H}_{11} \mathrm{ClNO}$ $\left([\mathrm{M}+\mathrm{H}]^{+}\right)$232.0524, found 232.0530 .

7-氯-2,4-二甲基呋喃[2,3-b]喹啉(2g)：白色固体, 32 mg, 产率 73\%. m.p. $150 \sim 153{ }^{\circ} \mathrm{C} ;{ }^{1} \mathrm{H}$ NMR $(600 \mathrm{MHz}$, $\left.\mathrm{CDCl}_{3}\right) \delta: 8.03(\mathrm{dd}, J=9.6,6.4 \mathrm{~Hz}, 1 \mathrm{H}), 7.68(\mathrm{dd}, J=10.4$, $2.8 \mathrm{~Hz}, 1 \mathrm{H}), 7.44(\mathrm{~s}, 1 \mathrm{H}), 7.30 \sim 7.25(\mathrm{~m}, 1 \mathrm{H}), 2.92(\mathrm{~s}$, $3 \mathrm{H}), 2.43(\mathrm{~s}, 3 \mathrm{H}) ;{ }^{13} \mathrm{C} \mathrm{NMR}\left(150 \mathrm{MHz}, \mathrm{CDCl}_{3}\right) \delta: 162.3$, $161.4,142.5,139.4,125.7,125.6,115.5,114.9,114.6$, 112.3 , 112.1, 14.5, 11.4. HRMS (ESI) calcd for $\mathrm{C}_{13} \mathrm{H}_{11} \mathrm{CINO}\left([\mathrm{M}+\mathrm{H}]^{+}\right)$232.0524, found 232.0532.

6-氯-2,4-二甲基呋喃[2,3-b]喹啉(2h): 白色固体, 36 mg, 产率 79\%. m.p. $175 \sim 177{ }^{\circ} \mathrm{C} ;{ }^{1} \mathrm{H}$ NMR $(400 \mathrm{MHz}$, $\left.\mathrm{CDCl}_{3}\right) \delta: 8.10(\mathrm{~d}, J=8.8 \mathrm{~Hz}, 1 \mathrm{H}), 8.07(\mathrm{~d}, J=2.0 \mathrm{~Hz}$, $1 \mathrm{H}), 7.64(\mathrm{dd}, J=8.8,2.4 \mathrm{~Hz}, 1 \mathrm{H}), 7.53(\mathrm{~s}, 1 \mathrm{H}), 2.95$ (s, $3 \mathrm{H}), 2.47$ (s, 3H); ${ }^{13} \mathrm{C}$ NMR (100 MHz, $\left.\mathrm{CDCl}_{3}\right) \delta: 161.1$, $156.4,143.6,130.5,129.9,129.7,122.7,120.6,116.4$, 115.6, 29.7, 14.5, 11.4. HRMS (ESI) calcd for $\mathrm{C}_{13} \mathrm{H}_{11} \mathrm{ClNO}$ $\left([\mathrm{M}+\mathrm{H}]^{+}\right)$232.0524, found 232.0524.

6-溴-2,4-二甲基呋喃[2,3-b]喹啉(2i): 白色固体, 44 $\mathrm{mg}$, 产率 79\%. m.p. $172 \sim 174{ }^{\circ} \mathrm{C} ;{ }^{1} \mathrm{H}$ NMR $(600 \mathrm{MHz}$, $\left.\mathrm{CDCl}_{3}\right) \delta: 8.24(\mathrm{~s}, 1 \mathrm{H}), 7.94(\mathrm{~d}, J=9.0 \mathrm{~Hz}, 1 \mathrm{H}), 7.74(\mathrm{~d}$, $J=9.0 \mathrm{~Hz}, 1 \mathrm{H}), 7.52$ (s, 1H), 2.94 (s, 3H), 2.47 (s, 3H); ${ }^{13} \mathrm{C} \mathrm{NMR}\left(150 \mathrm{MHz}, \mathrm{CDCl}_{3}\right) \delta: 161.9,143.5,143.4,137.9$, $131.9,130.7,127.3,126.0,120.4,118.2,115.5,14.3,11.5$. HRMS (ESI) calcd for $\mathrm{C}_{13} \mathrm{H}_{10} \mathrm{BrNNaO}\left([\mathrm{M}+\mathrm{Na}]^{+}\right)$ 297.9838, found 297.9837.

2-甲基-4-苯基呋喃[2,3- $b$ ]喹啉(2k)：白色固体，47 mg, 产率 91\%. m.p. $163 \sim 165{ }^{\circ} \mathrm{C} ;{ }^{1} \mathrm{H}$ NMR $(400 \mathrm{MHz}$, $\left.\mathrm{CDCl}_{3}\right) \delta: 8.20(\mathrm{~d}, J=8.4 \mathrm{~Hz}, 1 \mathrm{H}), 7.76 \sim 7.67(\mathrm{~m}, 2 \mathrm{H})$, 7.56 (dd, $J=6.4,3.2 \mathrm{~Hz}, 4 \mathrm{H}), 7.47 \sim 7.41$ (m, 3H), 1.73 (s, $3 \mathrm{H}) ;{ }^{13} \mathrm{C}$ NMR $\left(100 \mathrm{MHz}, \mathrm{CDCl}_{3}\right) \delta: 161.26,144.28$, $143.52,143.14,135.16,129.91,129.03,128.56,128.10$, $126.03,125.59,124.74,119.38,115.67,10.05$. HRMS (ESI) calcd for $\mathrm{C}_{18} \mathrm{H}_{14} \mathrm{NO}\left([\mathrm{M}+\mathrm{H}]^{+}\right)$260.1070, found 260.1070 .

辅助材料 (Supporting Information) 化合物的核磁谱 图. 这些材料可以免费从本刊网站 (http://sioc-journal.cn/)上下载. 


\section{Referenes}

[1] (a) Abass, M. Heterocycles 2005, 65, 901.

(b) Wang, R.; Liu, Z.-Q. Med. Chem. Res. 2013, 22, 1563.

(c) Huffman, J. W.; Browder, L. E. J. Org. Chem. 1964, 29, 2598.

[2] (a) Grougnet, R.; Magiatis, P.; Fokialakis, N.; Mitaku, S.; Skaltsounis, A. L.; Tillequin, F.; Sevenet, T.; Litaudon, M. J. Nat. Prod. 2005, 68, 1083.

(b) Ambrozin, A. R. P.; Mafezoli, J.; Vieira, P. C.; Fernandes, J. B.; da Silva, M.; Ellena, J. A.; de Albuquerque, S. J. Braz. Chem. Soc. 2005, 16, 434

(c) Ito, C.; Itoigawa, M.; Sato, A.; Hasan, C. M.; Rashid, M. A.; Tokuda, H.; Mukainaka, T.; Nishino, H.; Furukawa, H. J. Nat. Prod. 2004, 67, 1488.

(d) Ayafor, J. F.; Okogun, J. I. J. Chem. Soc., Perkin Trans. 1 1982, 909.

(e) Okogun, J. I.; Ayafor, J. F. Chem. Commun. 1977, 652.

[3] (a) Mabire, D.; Coupa, S.; Adelinet, C.; Poncelet, A.; Simonnet, Y.; Venet, M.; Wouters, R.; Lesage, A. S. J.; Van Beijsterveldt, L.; Bischoff, F. J. Med. Chem. 2005, 48, 2134.

(b) Michael, J. P. Nat. Prod. Rep. 2002, 19, 742.

(c) Michael, J. P. Nat. Prod. Rep. 2004, 21, 650.

(d) Michael, J. P. Nat. Prod. Rep. 2005, 22, 627.

(e) Michael, J. P. Nat. Prod. Rep. 2003, 20, 476.

[4] Tuppy, H.; Bohm, F. Angew. Chem., Int. Ed. 1956, 68, 388.

[5] (a) Yu, L.-Z.; Hu, X.-B.; Xu, Q.; Shi, M. Chem. Commun. 2016, 52, 2701.

(b) Boyd, D. R.; Sharma, N. D.; Barr, S. A.; Carroll, J. G.; Mackerracher, D.; Malone, J. F. J. Chem. Soc., Perkin Trans. 1 2000, 3397.

[6] (a) Aillaud, I.; Bossharth, E.; Conreaux, D.; Desbordes, P.; Monteiro, N.; Balme, G. Org. Lett. 2006, 8, 1113.

(b) Sekar, M.; Prasad, K. J. R. J. Nat. Prod. 1998, 61, 294.

(c) Narasimhan, N. S.; Mali, R. S. Tetrahedron 1974, 30, 4153.

(d) Collins, J. F.; Gray, G. A.; Grundon, M. F.; Harrison, D. M.; Spyropoulos, C. G. J. Chem. Soc., Perkin Trans. 1 1973, 94. (e) Narasimhan, N. S.; Paradkar, M. V.; Alurkar, R. H. Tetrahedron 1971, 27, 1351.

(f) Cooke, R. G.; Haynes, H. F. Aust. J. Chem. 1958, 11, 225.

(g) Grundon, M. F.; McCorkindale, N. J. J. Chem. Soc. 1957, 2177.

(h) Guo, R.-H.; Zhang, Q.; Ma, Y.-B.; Luo, J.; Geng, C.-A.; Wang, L.-J.; Zhang, X.-M.; Zhou, J.; Jiang, Z.-Y.; Chen, J.-J. Eur. J. Med. Chem. 2011, 46, 307.

[7] (a) Nagarajan, R.; Magesh, C. J.; Perumal, P. T. Synth. Stuttg. 2004 69.

(b) Yadav, J. S.; Reddy, B. V. S.; Madhuri, C. R.; Sabitha, G. Synth. Stuttg. 2001, 1065.

(c) Yadav, J. S.; Reddy, B. V. S.; Gayathri, K. U.; Prasad, A. R. Synth. Stuttg. 2002, 2537.

[8] Li, X.; Wang, C; Zheng, L. Chin. J. Org. Chem. 2006, 26, 1144 (in Chinese). (李雄武, 汪朝阳, 郑绿茵, 有机化学, 2006, 26, 1144.)

[9] (a) Zhang, Z.; Zhang, Q.; Sun, S.; Xiong, T.; Liu, Q. Angew. Chem., Int. Ed. 2007, 46, 1726.

(b) Ru, T. Light Ind. Sci. Technol. 2013, 5, 58 (in Chinese).

(茹婷婷, 轻工科技, 2013, 5,58 .)

(c) Du, W.; Curran, D. P. Org. Lett. 2003, 5, 1765.

[10] Zhang, Q.; Zhang, Z.; Yan, Z.; Liu, Q.; Wang, T. Org. Lett. 2007, 9, 3651.

[11] Zhang, Z. G. Ph.D. Dissertation, Northeast Normal University, Changchun, 2010 (in Chinese). (张志国，博士论文，东北师范大学，长春, 2010.)

[12] Sai, K. K. S.; Gilbert, T. M.; Klumpp, D. A. J. Org. Chem. 2007, $72,9761$.

[13] Heaney, H. In Comprehensive Organic Synthesis, Vol. 2, Eds.: Trost, B. M.; Fleming, I., Pergamon, Oxford, 1991, pp. 733 752 .

[14] (a) Zhang, Z.; Zhang, Q.; Yan, Z.; Liu, Q. J. Org. Chem. 2007, 72, 9808.

(b) Xiong, T.; Zhang, Q.; Zhang, Z.; Liu, Q. J. Org. Chem. 2007, 72,8005 .

(Cheng, F.) 